\title{
Liter per Day per Microgram per Meter Squared per Day
}

National Cancer Institute

\section{Source}

National Cancer Institute. Liter per Day per Microgram per Meter Squared per Day. NCI

Thesaurus. Code C120737.

A unit of concentration equal to liter per day divided by microg ram per meter squared per day. 